Research Article

\title{
FEASIBILITY OF GOLD BASED HALL DEVICES FOR BIOSENSING PURPOSES
}

\section{Davut IZCI ${ }^{*}$, John HEDLEY'}

\begin{abstract}
Hall sensors widely dominates the field of magnetic sensing. Basically, they produce information in terms of voltage with respect to applied field. The produced output depends on several parameters such as carrier mobility, carrier concentration, thickness and device geometries. In brief, materials with higher mobility and less thickness provides better candidates for such purposes. Looking at those parameters; gold-based Hall devices may not be thought of as the best candidate for magnetic field sensing. However, this does not suggest that it cannot be used for biosensing purposes. Gold presents an advantage of potential label-free biosensing device development since it can easily be functionalized for biosensing purposes. In this study, the feasibility of gold-based Hall devices was investigated through different fabrication techniques with and without adding separate layers including materials such as copper, nickel and chromium. The characterization has revealed that devices with smaller dimensions produce better output. The results showed that using gold to fabricate Hall sensors has merit for potential label-free biosensing purposes by designing a suitable geometry and following relevant microfabrication techniques.
\end{abstract}

Key words: Hall sensors, microfabrication, gold-based Hall devices

\section{Introduction}

Biosensors are analytical devices that are used in various fields such as food safety, environmental monitoring and medical diagnostics. A biosensor is combined of bioreceptor and transducer. Those two elements are integrated in such a way that the detection of specific target molecules are allowed. A bioreceptor is a specific recognition element for detecting desired chemicals and biomolecules whereas the transducer is used to convert the detection event into a meaningful description by means of electrical, mechanical or optical signals.

Historically, the field of biosensor research traces back to 1962 with enzyme electrode development by Lenard C. Clark [1] for testing glucose levels in blood and since then the bio-sensing field has been contributed by scientists from different research backgrounds. In 1970s, the first generation of biosensors was commercialized [2]. Further developments followed by describing a biorecognition system for the use of whole cell. Research and developments on the field has continued, particularly over the last decade. It is likely that the biosensors will have a huge impact on point of care diagnostics [3] because they have potential to be simplified and reduced in size, thus providing

\footnotetext{
${ }^{1}$ Vocational School of Technical Sciences, Batman University, Batman, Turkey, (davut.izci@batman.edu.tr)

(iD) https://orcid.org/0000-0001-8359-0875

${ }^{2}$ School of Engineering, Newcastle University, Newcastle upon Tyne, UK, (john.hedley@ newcastle.ac.uk)

(iD) https://orcid.org/0000-0002-2893-8776
} 
easy to use products. Therefore, this field needs to be improved based on new materials and design implementations to achieve a detection range of very small concentrations.

In order to detect a specific desired biological element, the sensor surface should be functionalised and this is usually done with complementary molecule [1]. In the presence of desired biological element, a capture process occurs. This process refers to the recognition step. A transducer is then used for converting the recognition event into a measurable electrical signal. A desired target molecule is captured by bio-receptor and this event creates a physical change in the transducer. The physical change can be measured as electrical, mechanical, optical or electrochemical based on transducer type. This change is converted to an electrical signal for a meaningful interpretation of the detection. The signal from output of the transducer is usually very small and needs to be amplified. In addition to amplification, any noisy harmonics should also be removed. Figure 1 represents a complete bio-sensing system depicting recognition (a), transducing (b), signal amplification (c) and processing (d) with recording/displaying (e) steps.

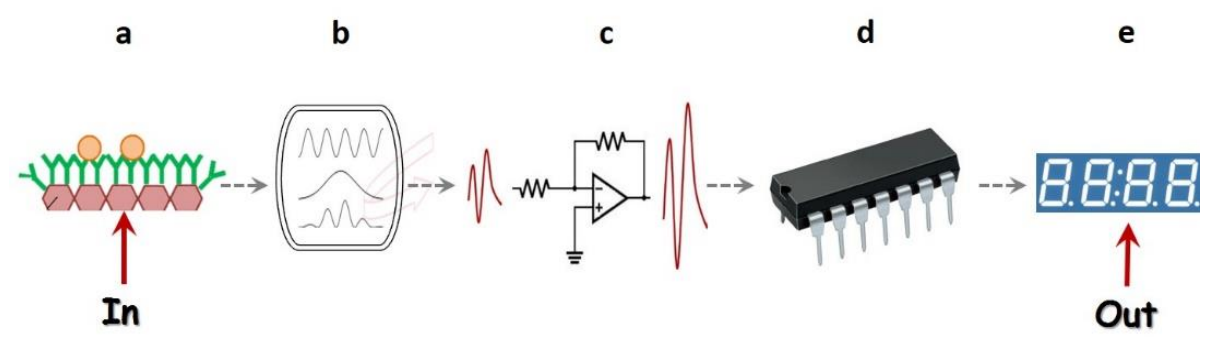

Figure 1. Recognition (a), conversion (b), signal amplification (c), processing (d), recording and displaying (e) steps.

Biosensors field is one of the fields that requires more improvements and currently an enormous amount of work is undertaken [4], [5]. Using magnetism is one of the techniques that was widely used to form detection platforms for biosensing purposes since it has a huge promise [6]-[8]. Apart from its key role in daily life from electric production to data storage and from quantitative explanation of physical properties of materials to particle acceleration [9], magnetic field has a huge potential of providing non-destructive and highly efficient detection platforms in biosensing field [6]. It provides a low intrinsic background in biological systems since those systems have no comparable biological signal [10]. Several mechanisms were reported based on different applications of magnetism such as magnetoresistance [11], planar Hall effect [12], spin valve [13], superconducting quantum interference devices (SQUID) [14], and Hall effect [15]. Amongst them, the Hall effect principle is the easiest way of achieving a magnetic sensor although it may not reach the sensitivity limits offered by some of those structures, e.g. SQUID sensors. Although each application has some certain advantages in terms of sensitivity, the specific requirements for operation such as low temperature demand in SQUID sensors, or fabrication complexity could make them unpractical. Thus, a considerable effort can be observed in biosensing applications that employ the Hall effect principle [16]-[18]. The Hall effect principle can briefly be explained as the transverse voltage that occurs due to Lorentz force. This force is appeared due to deviation in flow of electrons which can be observed under the presence of perpendicularly applied magnetic field. The obtained voltage depends on several parameters such as applied current and field, thickness and geometrical structure along with carrier mobility and density of a particular material.

Any thin layer of metal or semiconductor in a simple rectangular shape with four contacts could be considered as a Hall device although they would suffer from significant small potential difference produced at the output due to their electric transport parameters. Because, certain requirements such as 
carrier mobility, density of charge carriers etc., should be satisfied in materials to have effective and sensitive Hall devices. Several structures have been reported such as silicon, bismuth, indium antimonite thin films and two-dimensional electron gas heterostructures for fabrication of Hall devices [15]. Commercially available Hall sensors are dominated by silicon, due to well-developed CMOS manufacturing process [19]. However, in applications requiring higher sensitivity, heterostructures from III-V compounds are required because of superior electron transport properties they have [20]. In the latter type of devices, manufacturing cost can be high and integration with signal processing circuitry may be difficult [19]. Indium antimonite [21], bismuth [22] and graphene [23] thin films are also used in Hall applications due to the high electron mobility properties they retain and the linear response they demonstrate for a wide range of field strengths. However, gold based sensors have been designed and manufactured for this study as an alternative route. The aim was to explore a cheaper and easier way of implementation for Hall based biosensors as gold can easily be functionalised for biosensing purposes [24]-[26] if a label-free approach is implemented.

\section{Design}

Hall devices are basically devices that are producing information as an output voltage taking the applied field into account. Hall voltage output is proportional with carrier mobility and inversely proportional with carrier concentration, however, the geometrical structure has certain effects in terms of produced output [27]. Therefore, designing a suitable geometrical structure has a huge influence in terms of produced output. Scaling those structures are also affecting the output voltage [28]. Several devices with different geometrical structures and dimensions was designed for this study. Figure 2 is a sketch of basic geometrical structures with corresponding dimensions (in terms of length and width) on the masks. Active areas that were ranging from $1 \mathrm{~mm}$ to $3 \mathrm{~mm}$ were designed to be used for devices on FR4 substrate. The reason of designing devices with relatively large sizes (millimetre range) was due to PCB fabrication limits.

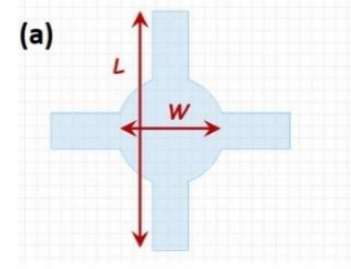

(c)

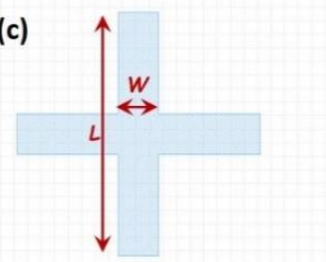

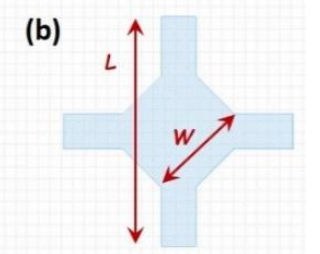

(d)

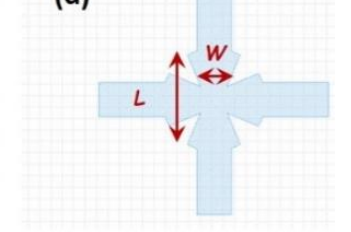

Figure 2. Representation of circle (a), square (b), cross (c) and cauliflower (d) geometries (L: Length, W: Width).

A second design including cross structures with relatively smaller sizes $(500 \mu \mathrm{m})$ was also designed for this study. The second design was used to operate laser machine (HPC Laserscript) to create masks for gold sputtering on a glass substrate. The reason of choosing the latter dimension was because of the limit that the laser cutting machine can reach to obtain a clear mask from acrylic. Due to thermal distortion in acrylic, smaller dimensions with clear shapes were not feasible.

To fabricate micro-scale gold devices on a silicon substrate, a mask was designed with several geometrical forms along with various length to width ratios $(l / w)$ greater than 3 , in order to avoid 
weakening the Hall effect due to geometrical factor [15], and made on a 4-inch quartz to form devices having active areas of $10 \mu \mathrm{m}, 20 \mu \mathrm{m}, 40 \mu \mathrm{m}$ and $60 \mu \mathrm{m}$. Figure 3 shows the designed mask for the latter purpose.

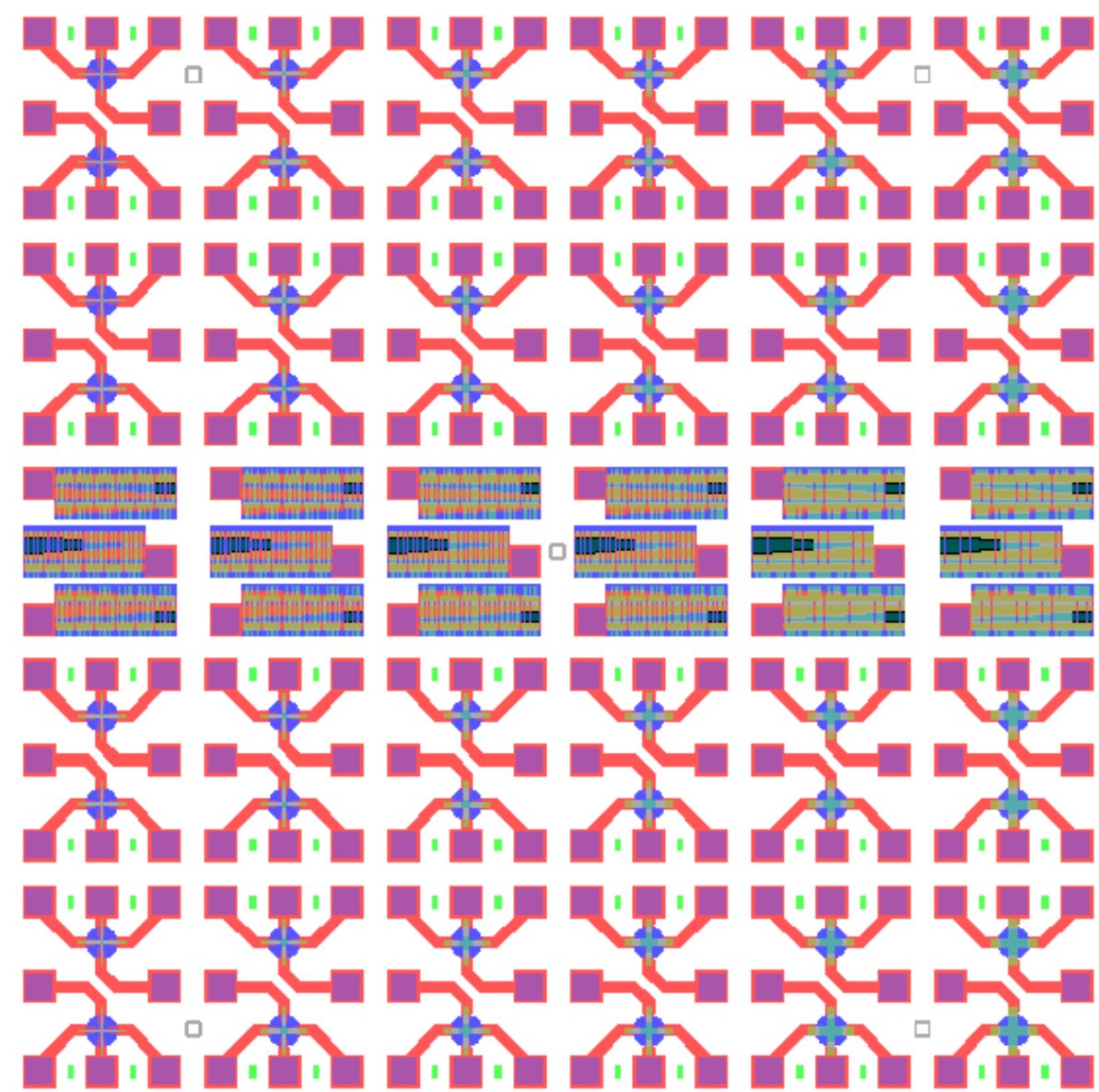

Figure 3. Designed mask with Hall device structures from 10 microns to 60 microns.

\section{Materials}

Thin films [29], 2DEG heterostructures [30] or single layer atomic structures such as graphene and its derivatives [31] are the excellent choices since they meet the required criteria for better Hall devices. However, working with those materials requires familiarity of microfabrication processes which could be expensive to a certain extent and could cost time. Therefore, this study focused on devices that are relatively easy to fabricate. Three different routes were followed to manufacture gold based Hall devices. Firstly, gold devices, in combination with nickel and copper, were used to be made on an FR4 substrate following traditional PCB fabrication routes. Secondly, pure gold structures were employed to be directly used on a glass substrate. This approach was simple in terms of fabrication with feasibility as Hall sensors. The last approach was to use gold based micro-Hall devices in combination of chromium on a silicon die by implementing microfabrication steps. This method of fabrication was more complicated to fabricate devices and leading to slightly less sensitive sensors for relatively bigger sizes, yet, can be applied to biosensing applications. 


\section{Fabrication}

\subsection{Fabrication on FR4 Substrate}

In this approach, the aim was to use an alternative and easier way of device fabrication and practically observe the response of an inhomogeneous combination of metals along with different shapes and sizes. The devices were made of single-sided PCB with no plated-through holes and were built on $1.6 \mathrm{~mm}$ FR4 epoxy glass fibre substrate along with three layers of different materials using PCB fabrication techniques. It was consisted of $35 \mu \mathrm{m}$ of copper $(\mathrm{Cu})$ layer, 3-5 $\mu \mathrm{m}$ nickel $(\mathrm{Ni})$ layer, and $0.3 \mu \mathrm{m}$ of gold $(\mathrm{Au})$ layer. A cross-sectional view of the design is given in Figure 4 .

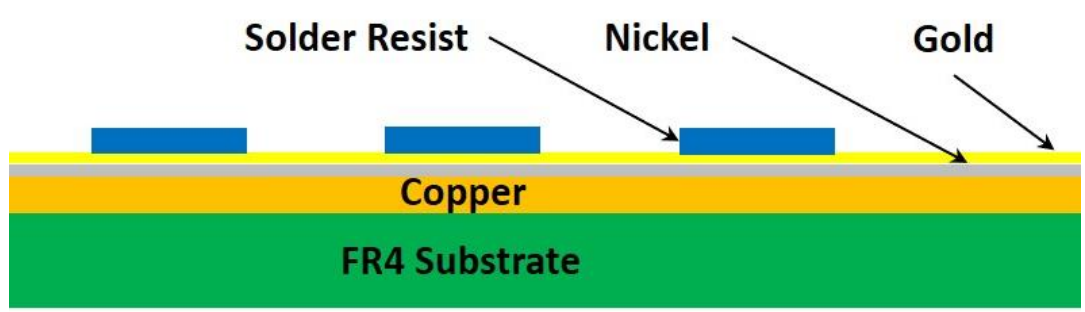

Figure 4. Cross-sectional view of designed Hall devices on PCB.

Several devices with different geometrical structures (Greek cross, square, cauliflower, round etc.,) and dimensions ranging from $1 \mathrm{~mm}$ to $3 \mathrm{~mm}$ were fabricated for each shape - see Figure 5 . The reason of designing devices with relatively large sizes was due to PCB fabrication limits. Most of the devices consisted of 4 contact points; 2 for biasing and other 2 for reading, respectively. The devices with 6 contacts (Figure 5(e) and Figure 5(f)) were aimed to be used for quantum Hall effect or magnetoresistance effects for future works. The areas covered by solder resist was designed for potentially limiting any bead attachment to the required areas of devices meaning that the functionalisation process can be performed only on active areas of the devices.

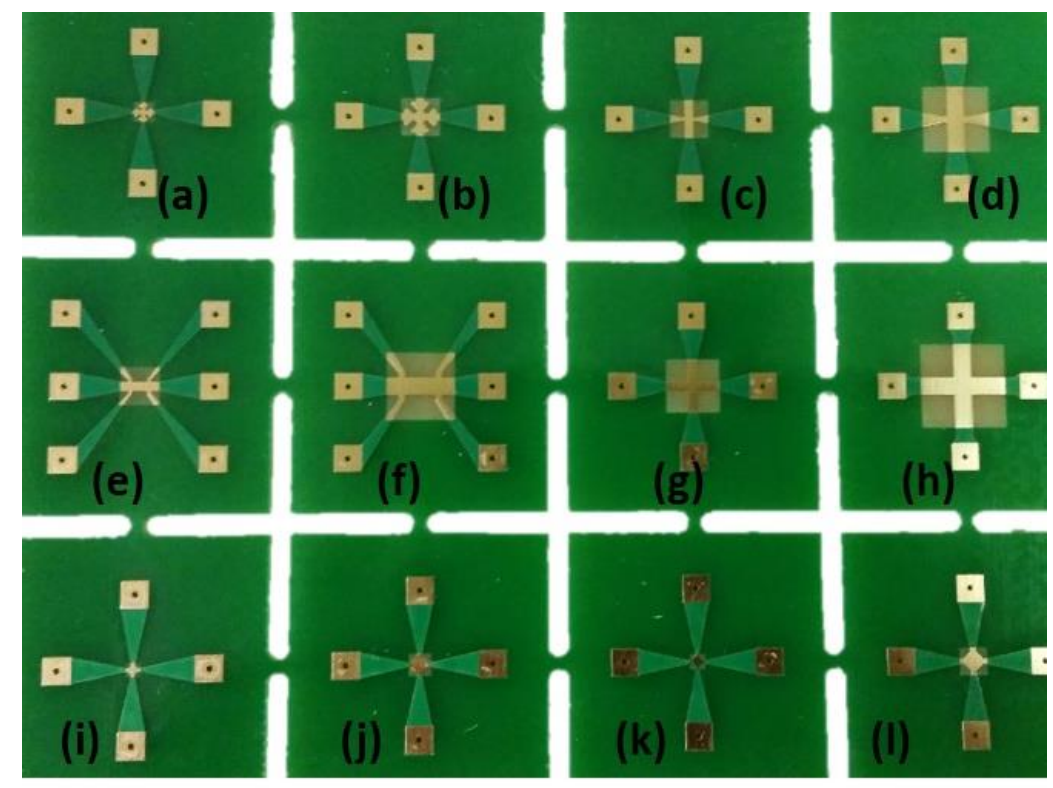

Figure 5. Actual view of fabricated Hall devices on an FR4 substrate. 


\subsection{Fabrication on Glass Substrate}

Devices that are explained in previous section had non-homogeneous structures since they included three different layers of materials. Additionally, surface roughness was not as smooth as desired. Therefore, additional structures were designed and made on a thin acrylic mask by cutting relevant structures with laser cutting machine. Then a gold layer of around $30 \mathrm{~nm}$ were formed by sputtering technique using a Bio-Rad Microscience Division SC500 sputter machine. Those devices had relatively large sizes ( $500 \mu \mathrm{m}$ and more) due to the limitation in laser beam size and thermal distortion issue in the acrylic. Figure 6 shows the sputter machine used for forming gold structures (a) along with the created acrylic masks on glass slides (b) and one of the slides with formed Hall devices (c).

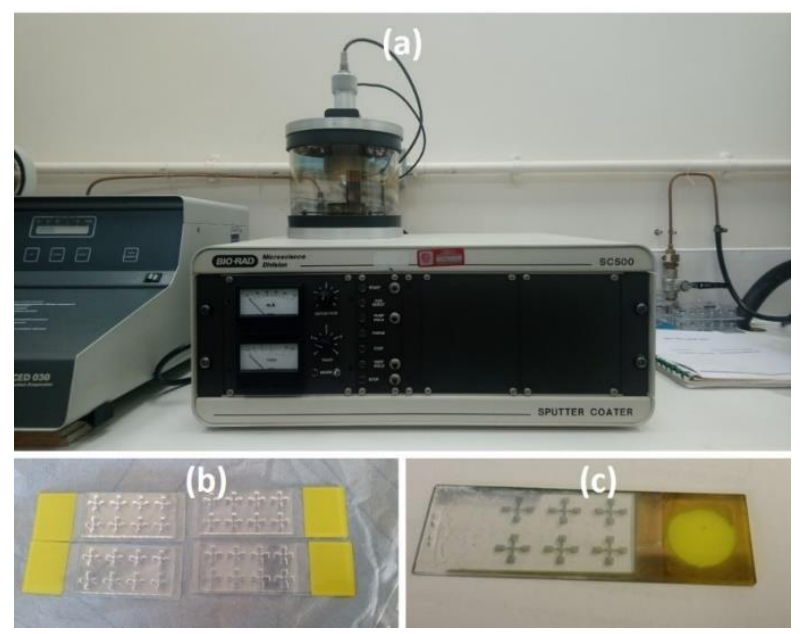

Figure 6. Gold sputter coater (a) with designed acrylic masks (b) and actual fabricated devices on glass substrate (c).

\subsection{Fabrication on Si Substrate}

This approach was implemented to fabricate gold devices with an adhesion layer. Micro-Hall devices from $10 \mu \mathrm{m}$ to $60 \mu \mathrm{m}$ were also fabricated using microfabrication techniques including lithography and lift-off processes by employing a $\mathrm{SiO}_{2} / \mathrm{Si}$ substrate. A chromium layer was used to aid the adhesion. Figure 7 demonstrates the microfabrication steps designed for device manufacturing. 
(a)

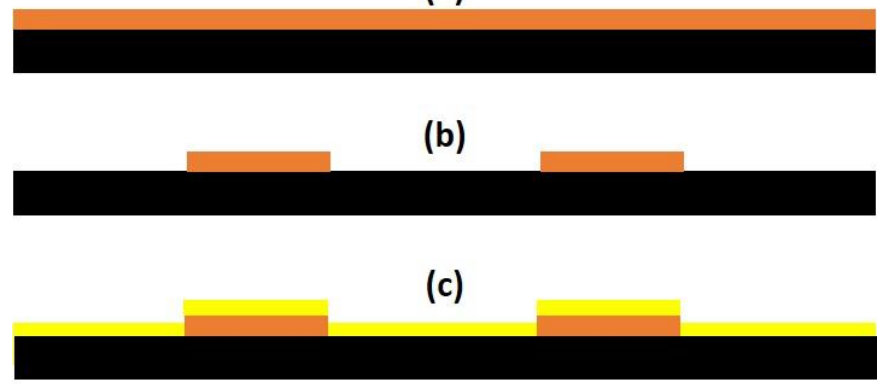

(d)

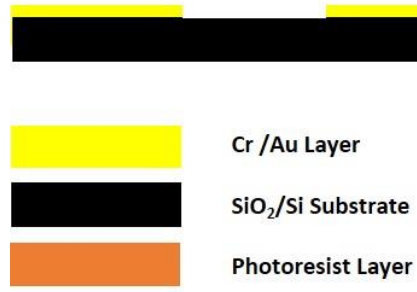

Figure 7. Lithography and lift-off process for micro-Hall gold devices. Positive photoresist cover (a), lithography and developing resist stages for constructing the pattern (b), chromium (Cr) and gold (Au) evaporation using e-beam evaporator (c), and lift-off process (d).

The fabrication started with cutting silicon substrates of around $10 \times 10 \mathrm{~mm}^{2}$ and cleaned before any process. The cleaning procedure took place initially by doing ultrasonic bath at $80{ }^{\circ} \mathrm{C}$ using NMP (N-methyl-2-pyrrolidone) and IPA (propan-2-ol) for 10 and 5 minutes, respectively, followed by rinsing with ultra-pure water. This was carried out as an initial step for organic clean. Then, further cleaning process was taken place using piranha solution (a mixture of $\mathrm{H}_{2} \mathrm{O}_{2}$ (hydrogen peroxide) and $\mathrm{H}_{2} \mathrm{SO}_{4}$ (sulphuric acid)) to remove any residue. The last step in cleaning was to employ RCA cleaning method (steps including $\mathrm{NH}_{4} \mathrm{OH}$ (ammonium solution), $\mathrm{H}_{2} \mathrm{O}_{2}$ and $\mathrm{BHF}$ (buffered hydrofluoric acid)). After wet-chemical cleaning procedure, the substrate was dried using nitrogen gun. The substrate was covered with negative photoresist (AZ5214E) by using a spin coater (EMS 6000 spin coater) and prebaked at $90{ }^{\circ} \mathrm{C}$ for 10 minutes, following the cleaning procedure. Then, the lithography process was performed with the aid of patterned glass mask. The sample was exposed to UV for 4 seconds using KarlSuss MJB-3 mask aligner. A post-bake stage took place by using a hot plate (Electronic Micro Systems Ltd Model 1000-1 Precision Hot Plate) at $120{ }^{\circ} \mathrm{C}$ for 30 seconds followed by a blank UV exposure for 8 seconds. The latter process was performed to use the photoresist as an image reversal. After lithography, patterns were obtained by developing the photoresist using AZ 326 MIF developer. A chromium layer of $7 \mathrm{~nm}$ and gold layer of $35 \mathrm{~nm}$ were evaporated using e-beam evaporator (Edwards 306 e-beam evaporator) after developing relevant structure. These thicknesses were decided to be adequate for visibility and adhesion. In addition, the stated thicknesses were decided to be sufficient for potential biosensing applications in liquid medium. The last step was to do lift-off process in order to remove unnecessary parts. Figure 8 shows the obtained device structures after following the steps explained above. 

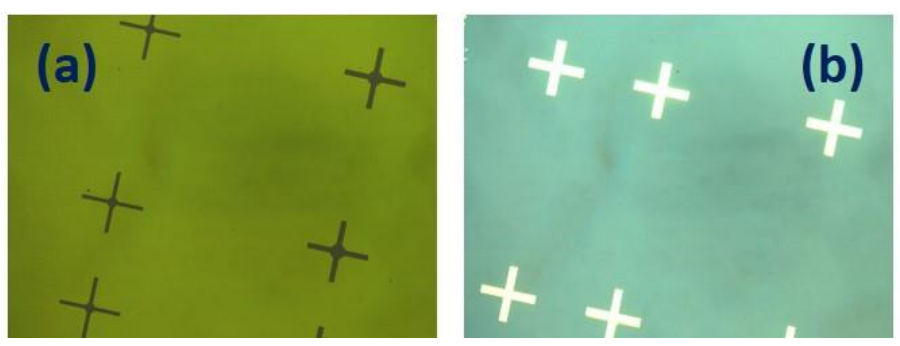

Figure 8. Patterned photoresist after lithography and developer (a), obtained device structures after lift-off (b).

A similar process was carried out to form contacts on the obtained structures. Meanwhile, it is worth to note that the steps given in Figure 8 are for forming the Hall features using negative photoresist. To obtain contacts, positive resist was used instead of negative resist due to feature arrangements on the mask. Therefore, the process took place in the reverse meaning that the features were formed on the areas where exposed to UV directly. In this case, the same photoresist was used, however, it was pre-baked at $90{ }^{\circ} \mathrm{C}$ for 15 minutes and the exposure time was 4 seconds without performing post-bake and blank exposure. The same developer was used to develop the resist and a layer of chromium with $30 \mathrm{~nm}$ along with a layer of gold with $250 \mathrm{~nm}$ were evaporated using e-beam evaporator. The fabrication was completed by doing the second lithography. Figure 9 shows the developed features aligned with previously formed Hall structures (a) and the devices with contacts after lithography process (b) along with the fabricated devices on a $10 \times 10 \mathrm{~mm}^{2}$ die (c).
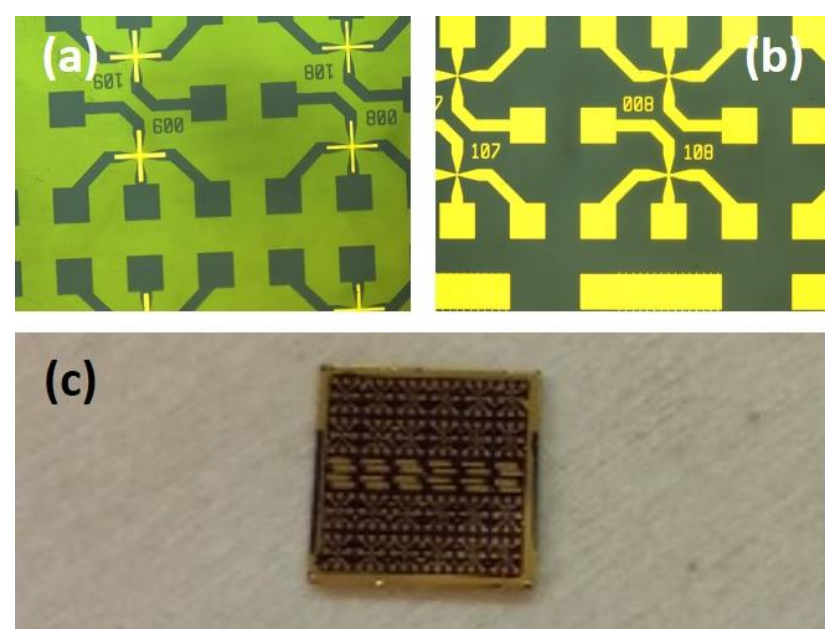

Figure 9. Patterning and forming contacts along with a silicon die used as a substrate with the fabricated devices on.

\section{Measurements, Results and Discussion}

\subsection{Devices on FR4 Substrate}

Devices on FR4 substrate had non-homogeneous material combination along with relatively thick and large structures. Initially, a low noise linear DC power supply (Farnell E30-28T) was used to drive the devices. The reason of not using a switching power supply was to minimize any noise caused by biasing source. Apart from this power supply, a Keithley 6221 AC and DC current source was also used for operating devices. To allow current flow, the contacts of the device were soldered using a screened cable. The reason of using the screened cable was to eliminate external noise effects. The PCB Hall device was placed in between designed c-core structure as shown in Figure 10 in order to apply a perpendicular magnetic field. 


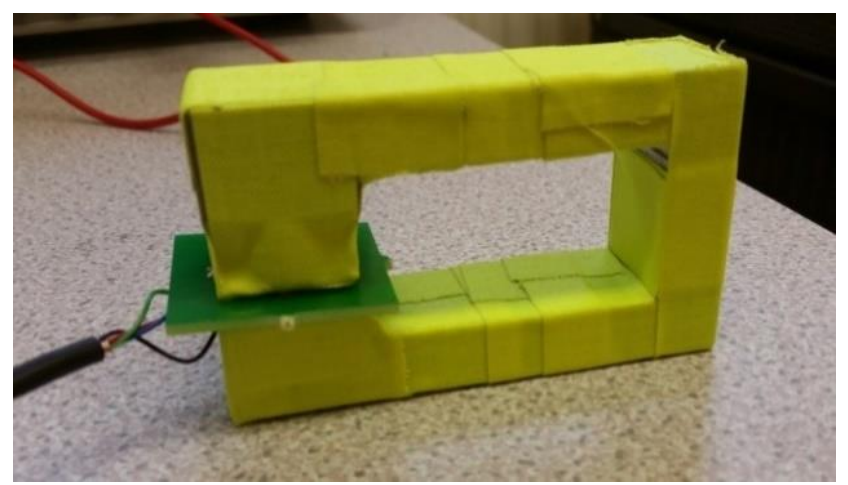

Figure 10. Performing Hall measurements using a designed structure consisting of a rare earth magnet and pure iron.

A custom made integrated circuitry [10] was used to perform measurements. The output voltages in few hundreds of nanovolts were obtained from PCB based Hall devices. Figure 11 presents a typical output obtained from gold devices on an FR4 substrate. A current-related sensitivity of $3 \mu \mathrm{V} /$ AT was calculated which proved that the sensitivity of those type of Hall sensors are not in a desired range as was estimated due to its relatively large and thick structure along with its non-homogeneous structure.

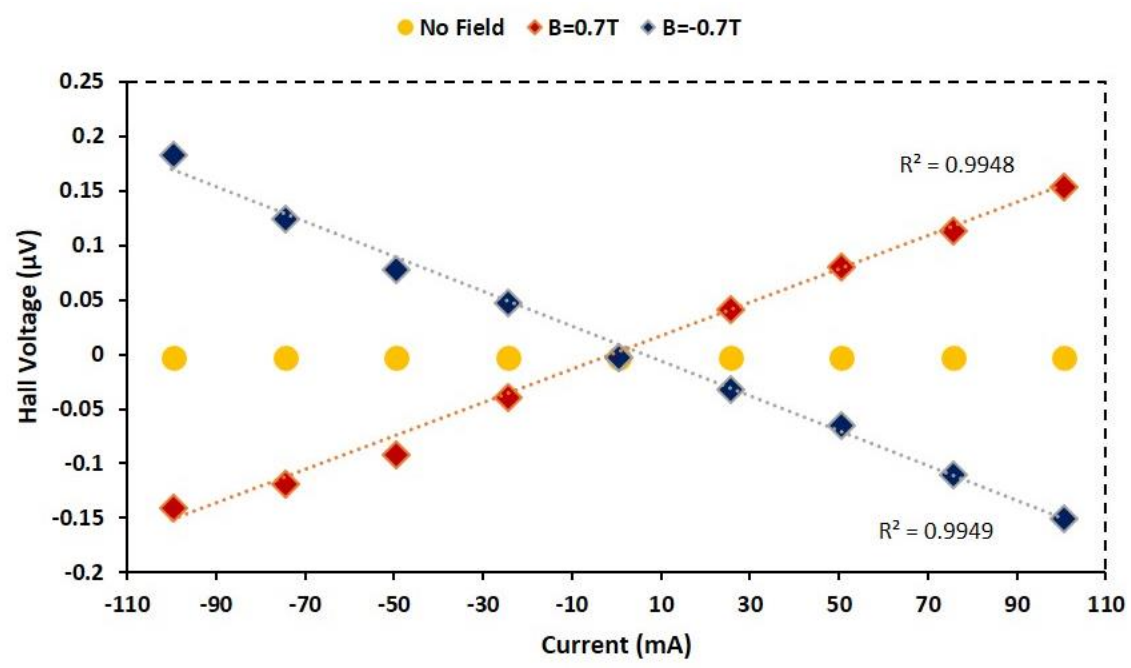

Figure 11. A typical Hall output from gold devices on FR4 substrate.

\subsection{Devices on Glass Substrate}

The gold devices on a glass substrate were designed and fabricated to eliminate the nonhomogeneous structure issue so that the sensitivity could be improved. To perform measurements, a screened cable was connected to the device's contacts with the aid of silver paint (RS Pro Silver Conductive Adhesive Paint). Similar to the case in the devices on FR4 substrate, the magnetic field was provided using the same iron core and rare earth magnet as shown in Figure 12. 


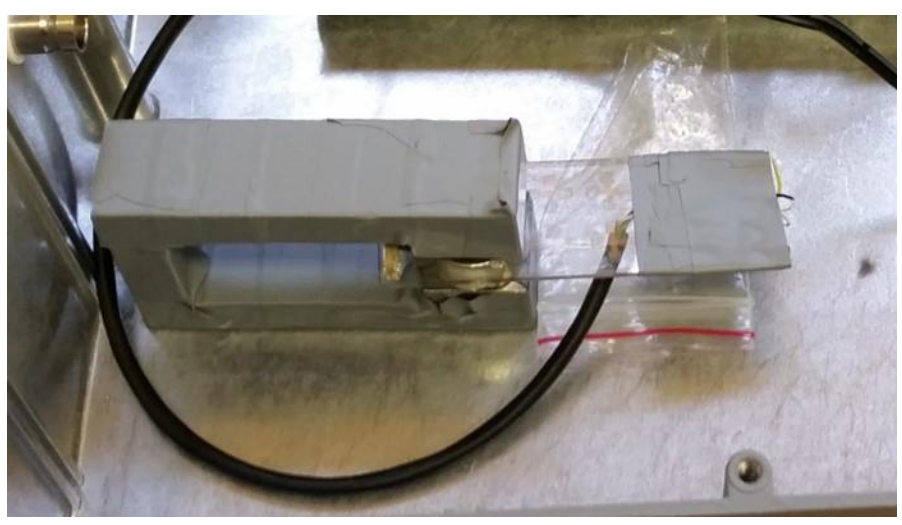

Figure 12. Au Hall device on glass substrate placed in magnetic field.

As was expected, this type of devices showed better sensitivity characteristics compared to devices on FR4. Because, it did not include different material combination and had thinner gold layer. They also presented a good linearity behaviour. Outputs in microvolt range were obtained from those devices as shown in Figure 13. The obtained values were better than the devices on FR4 substrate by about an order of magnitude. The behaviour they presented suggests that they can be adopted for applications where high sensitivity is not required. A current-related sensitivity of $5 \mathrm{mV} / \mathrm{AT}$ was obtained which confirms the improvement of the performance comparing FR4 based Hall sensors. However, in terms of biosensing applications, they are not suitable due to lack of adhesion to the substrate which consequently causes delamination when a liquid inserted on them.

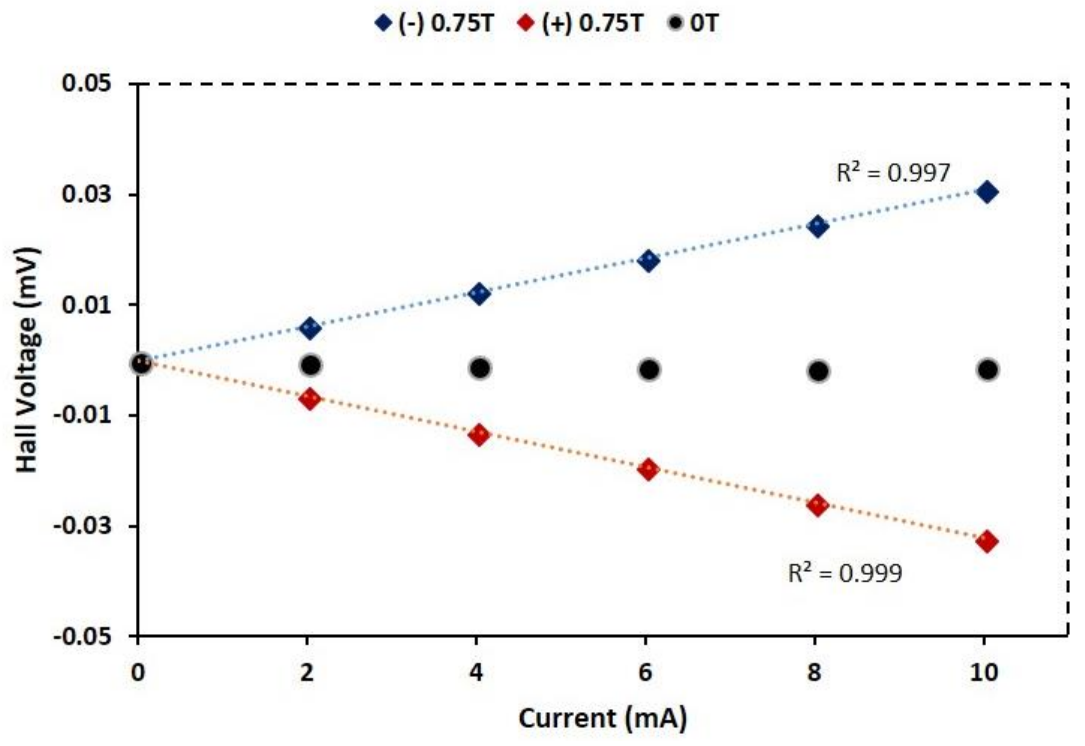

Figure 13. Response of a Au Hall device on glass substrate to magnetic fields under varying current.

\subsection{Devices on Si Substrate}

Micro-fabricated gold devices were employed as an alternative to gold-based Hall devices on glass substrate to improve the adhesion. To do so, a layer of chromium was used, however, addition of this extra layer means that the performance of the device would decrease. To overcome this issue, the size of the sensors was decreased to compensate the reduction of the performance due to adhesion layer. The fabricated devices were wire-bonded to a 28-pin DIP chip, as demonstrated in Figure 14, using a wire bonder (Kulicke \& Soffa Industries Model 4700 Wire Bonder). 

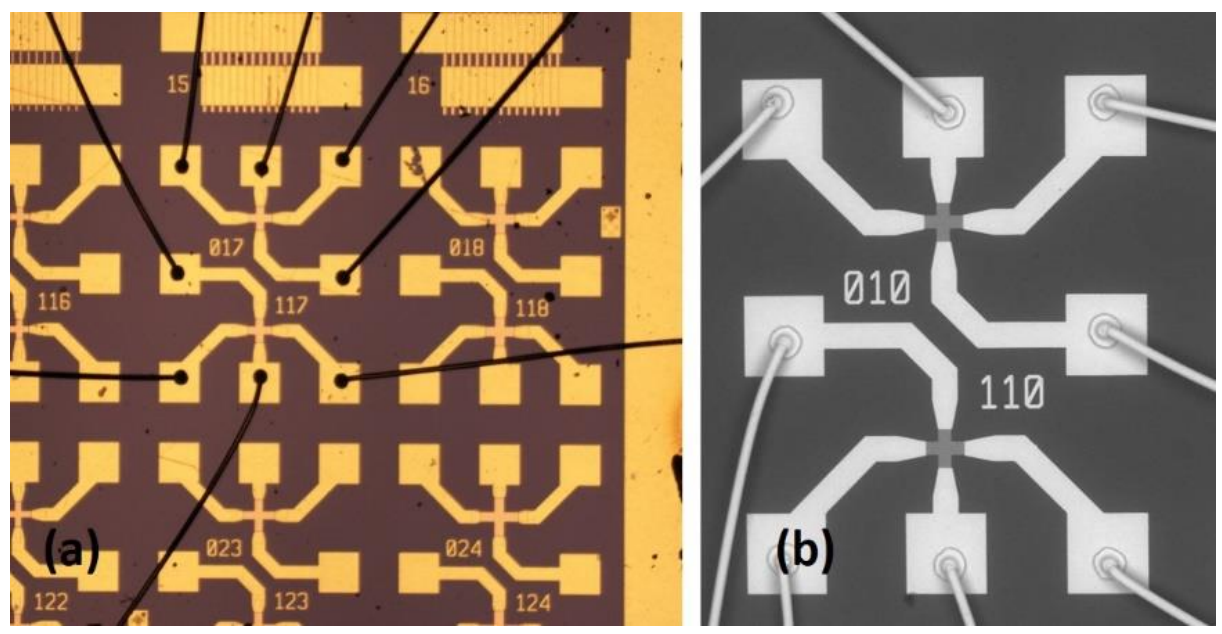

Figure 14. An optical (a) and SEM (b) image of wire-bonded contacts to a chip.

The initial measurements were performed on devices with $60 \mu \mathrm{m}$ sizes. The performance parameters with those devices were no better than gold devices on glass substrate. This was an expected reduction since micro-Hall gold devices were fabricated with an additional layer of chromium. A current-related sensitivity of $3 \mathrm{mV} / \mathrm{AT}$ was obtained from those devices which verified the reduction in performance. Figure 15 shows the output change of a $60 \mu \mathrm{m}$ device with respect to magnetic field.

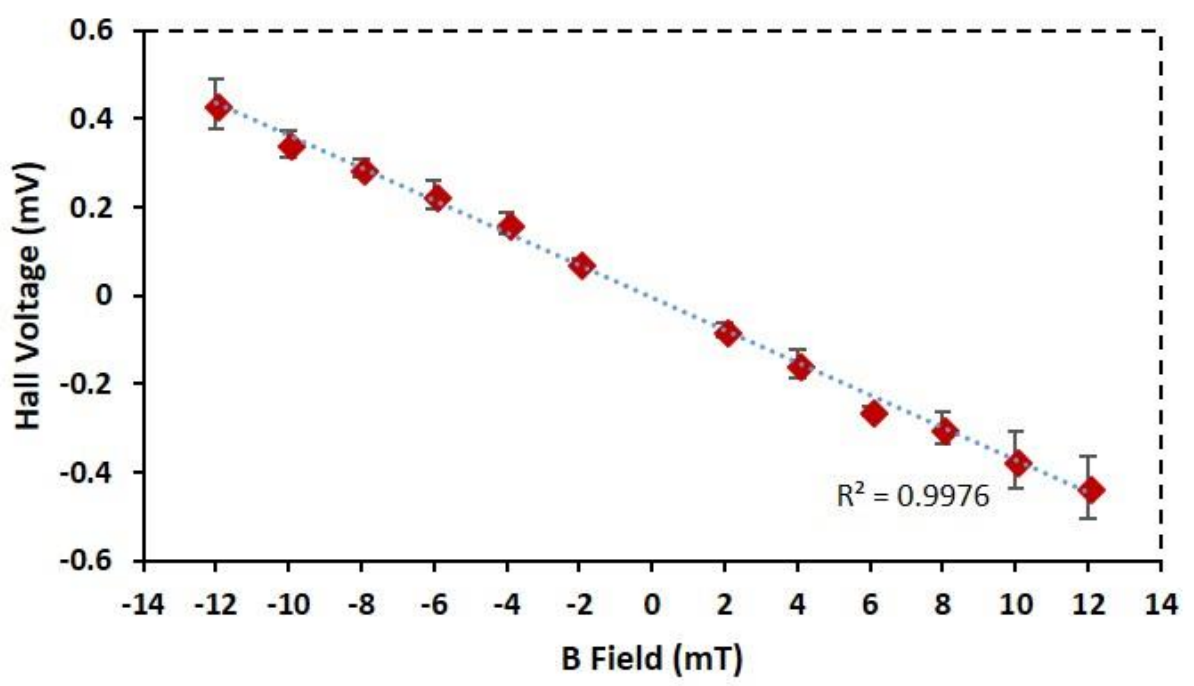

Figure 15. The output of a $\mathrm{Cr} / \mathrm{Au}$ device on silicon substrate with $60 \mu \mathrm{m}$ active area.

However, the following measurements on devices with $10 \mu \mathrm{m}$ active areas presented a considerably better performance. This was attributed to the size effect [28] meaning that the smaller the size the better the performance. A current-related sensitivity of $27 \mathrm{mV} / \mathrm{AT}$ was calculated for those devices. In Figure 16, the output characteristic of a $10 \mu \mathrm{m}$ device is presented with respect to varying current (a) and magnetic field (b). The device output with respect to varying current for positive and negative magnetic fields was demonstrated to be highly linear and repeatable. Similarly, the output of the device with respect to varying magnetic field with a costant biasing current has also showed promising figures. 

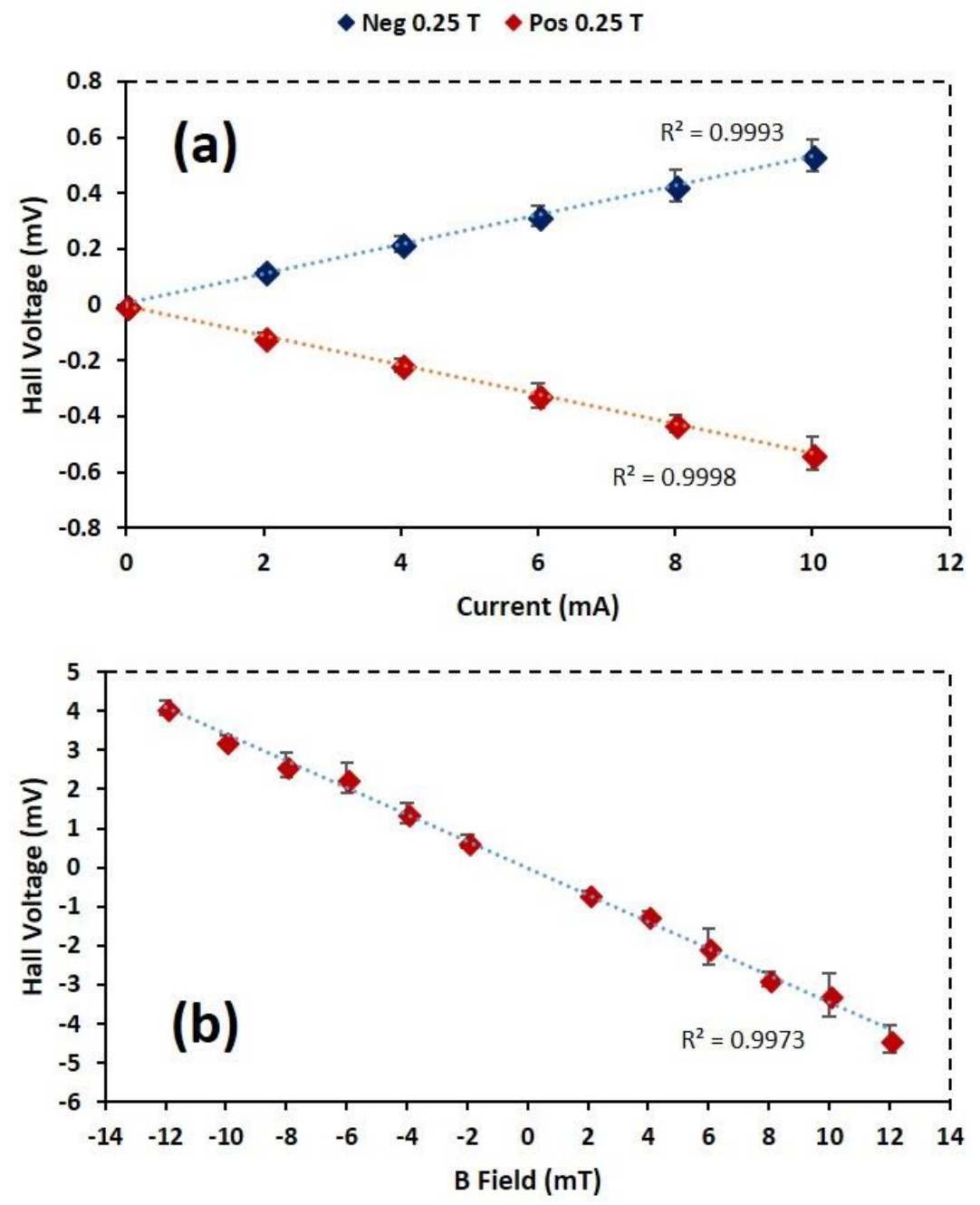

Figure 16. The output of a Cr/Au device on silicon substrate with $10 \mu \mathrm{m}$ active area.

The performance parameters for gold based devices manufactured via different routes is summarized in Table 1. As can be seen, eliminating non-homogeneous structure, reducing layer number and the size of the device has significant effect on performance parameters.

Table 1. Comparison of performance parameters for gold-based devices

\begin{tabular}{cc}
\hline \multicolumn{1}{c}{ Devices } & Current-related Sensitivity \\
\hline $\mathrm{Au} / \mathrm{Cu} / \mathrm{Ni}$ on PCB $(1 \mathrm{~mm}$ size $)$ & $3 \mu \mathrm{AT}$ \\
\hline $\mathrm{Au}$ on Glass Substrate $(500 \mu \mathrm{m}$ size $)$ & $5 \mathrm{mV} / \mathrm{AT}$ \\
\hline $\mathrm{Au} / \mathrm{Cr}$ on $\mathrm{SiO}_{2} / \mathrm{Si}$ Substrate $(60 \mu \mathrm{m}$ size $)$ & $3 \mathrm{mV} / \mathrm{AT}$ \\
\hline $\mathrm{Au} / \mathrm{Cr}$ on $\mathrm{SiO}_{2} / \mathrm{Si}$ Substrate $(10 \mu \mathrm{m}$ size $)$ & $27 \mathrm{mV} / \mathrm{AT}$ \\
\hline
\end{tabular}

\section{Conclusion}

In general, gold-based Hall devices on FR4 substrate cannot be considered for high sensitivity applications due to their electric material properties and non-homogeneous structure. However, reducing the layer sizes has merit for applications not requiring high sensitivity as was shown with gold devices on glass substrate. In reality, the performance parameters for the latter type of Hall sensors can significantly be improved if they can be made in smaller sizes as confirmed by gold devices on silicon substrate with $10 \mu \mathrm{m}$ active areas. In this work, the aim was to discover a cheaper and easier fabrication method, thus, the devices on glass substrate were made with larger sizes, due to 
limitation of laser size and distortion in acrylic because of thermal heat effect. Moreover, sputtering technique is not convenient for creating a smooth surface as it is bombarding the gold all around the surface. Therefore, employing an appropriate glass mask with smaller features and implementing lithographical processes would allow smaller sizes. Using electron beam evaporation can improve the surface smoothness which will consequently lead to devices with much better performances. Meanwhile, it is worth to note that creating devices with only a thin gold layer will not be suitable for biosensing applications although they might be used for applications requiring dry air. Because, the material is removed from the surface when it is introduced in liquid environment. This is due to the lack of the adhesion layer. In the meantime, employing an adhesion layer will cause distortion in the performance as was shown by $\mathrm{Cr} / \mathrm{Au}$ devices with $60 \mu \mathrm{m}$ active sizes. Nevertheless, this issue can be overcome by decreasing the feature size as was confirmed by $\mathrm{Cr} / \mathrm{Au}$ devices with $10 \mu \mathrm{m}$ active areas.

\section{References}

[1] S. P. Mohanty and E. Koucianos, "Biosensors: A tutorial review," IEEE Potentials, vol. 25, no. 2, pp. 35-40, 2006, doi: 10.1109/MP.2006.1649009.

[2] S. Song, H. Xu, and C. Fan, "Potential diagnostic applications of biosensors: Current and future directions," Int. J. Nanomedicine, vol. 1, no. 4, pp. 433-440, 2006, doi: 10.2147/nano.2006.1.4.433.

[3] S. K. Vashist, "Point-of-care diagnostics: Recent advances and trends," Biosensors, vol. 7, no. 4. 2017, doi: 10.3390/bios7040062.

[4] S. Vigneshvar, C. C. Sudhakumari, B. Senthilkumaran, and H. Prakash, "Recent advances in biosensor technology for potential applications - an overview," Front. Bioeng. Biotechnol., vol. 4, no. FEB, p. 11, 2016, doi: 10.3389/fbioe.2016.00011.

[5] B. Wang, U. Akiba, and J. I. Anzai, "Recent progress in nanomaterial-based electrochemical biosensors for cancer biomarkers: A review," Molecules, vol. 22, no. 7, p. 1048, 2017, doi: 10.3390/molecules22071048.

[6] D. Issadore et al., "Magnetic sensing technology for molecular analyses," Lab Chip, vol. 14, no. 14, pp. 2385-2397, 2014, doi: 10.1039/c4lc00314d.

[7] T. Takamura, P. J. Ko, J. Sharma, R. Yukino, S. Ishizawa, and A. Sandhu, "Magnetic-particlesensing based diagnostic protocols and applications," Sensors (Switzerland), vol. 15, no. 6, pp. 12983-12998, 2015, doi: 10.3390/s150612983.

[8] J. Llandro, J. J. Palfreyman, A. Ionescu, and C. H. W. Barnes, "Magnetic biosensor technologies for medical applications: A review," Med. Biol. Eng. Comput., vol. 48, no. 10, pp. 977-998, 2010, doi: 10.1007/s11517-010-0649-3.

[9] R. S. Popović, Hall effect devices, 2nd ed. Philadelphia : Institute of Physics Pub., 2004.

[10] D. İzci, "Constructing an Electronic Circuitry for Label-free Hall Biosensors," Balk. J. Electr. Comput. Eng., vol. 7, no. 4, pp. 366-372, Oct. 2019, doi: 10.17694/bajece.633908.

[11] G. Rizzi, F. W. Osterberg, A. D. Henriksen, M. Dufva, and M. F. Hansen, "On-chip magnetic bead-based DNA melting curve analysis using a magnetoresistive sensor," J. Magn. Magn. Mater., vol. 380, pp. 215-220, 2015, doi: 10.1016/j.jmmm.2014.09.004.

[12] M. Volmer and M. Avram, "Using permalloy based planar hall effect sensors to capture and detect superparamagnetic beads for lab on a chip applications," J. Magn. Magn. Mater., vol. 381, pp. 481-487, 2015, doi: 10.1016/j.jmmm.2014.10.172.

[13] T. Q. Hung, S. Oh, J. R. Jeong, and C. G. Kim, "Spin-valve planar Hall sensor for single bead detection," Sensors Actuators, A Phys., vol. 157, no. 1, pp. 42-46, 2010, doi: 10.1016/j.sna.2009.11.033.

[14] D. Drung et al., "Highly sensitive and easy-to-use SQUID sensors," IEEE Trans. Appl. Supercond., vol. 17, no. 2, pp. 699-704, 2007, doi: 10.1109/TASC.2007.897403.

[15] D. Izci, C. Dale, N. Keegan, and J. Hedley, "The Construction of a Graphene Hall Effect Magnetometer," IEEE Sens. J., vol. 18, no. 23, pp. 9534-9541, Dec. 2018, doi: 10.1109/JSEN.2018.2872604.

[16] A. Sandhu, Y. Kumagai, A. Lapicki, S. Sakamoto, M. Abe, and H. Handa, "High efficiency 
Hall effect micro-biosensor platform for detection of magnetically labeled biomolecules," Biosens. Bioelectron., vol. 22, no. 9-10, pp. 2115-2120, 2007, doi: 10.1016/j.bios.2006.09.021.

[17] K. Togawa et al., "Detection of magnetically labeled DNA using pseudomorphic AlGaAs/InGaAs/GaAs heterostructure micro-Hall biosensors," J. Appl. Phys., vol. 99, no. 8, 2006, doi: 10.1063/1.2162041.

[18] G. Mihajlović et al., "Detection of single magnetic bead for biological applications using an InAs quantum-well micro-Hall sensor," Appl. Phys. Lett., vol. 87, no. 11, 2005, doi: $10.1063 / 1.2043238$.

[19] H. Xu et al., "Batch-fabricated high-performance graphene Hall elements," Sci. Rep., vol. 3, p. 1207, 2013, doi: 10.1038/srep01207.

[20] M. Bando et al., "High sensitivity and multifunctional micro-Hall sensors fabricated using InAlSb/InAsSb/InAlSb heterostructures," J. Appl. Phys., vol. 105, no. 7, p. 07E909, 2009, doi: 10.1063/1.3074513.

[21] O. Kazakova et al., "Detection of a micron-sized magnetic particle using insb hall sensor," IEEE Trans. Magn., vol. 45, no. 10, pp. 4499-4502, 2009, doi: 10.1109/TMAG.2009.2025513.

[22] D. Petit, D. Atkinson, S. Johnston, D. Wood, and R. P. Cowburn, "Room temperature performance of submicron bismuth Hall probes," IEE Proc. Sci. Meas. Technol., vol. 151, no. 2, pp. 127-130, 2004, doi: 10.1049/ip-smt:20040167.

[23] B. Chen, L. Huang, X. Ma, L. Dong, Z. Zhang, and L. M. Peng, "Exploration of sensitivity limit for graphene magnetic sensors," Carbon N. Y., vol. 94, pp. 585-589, 2015, doi: 10.1016/j.carbon.2015.07.040.

[24] J. M. Pingarrón, P. Yáñez-Sedeño, and A. González-Cortés, "Gold nanoparticle-based electrochemical biosensors," Electrochim. Acta, vol. 53, no. 19, pp. 5848-5866, 2008, doi: 10.1016/j.electacta.2008.03.005.

[25] K. Saha, S. S. Agasti, C. Kim, X. Li, and V. M. Rotello, "Gold nanoparticles in chemical and biological sensing," Chem. Rev., vol. 112, no. 5, pp. 2739-2779, 2012, doi: $10.1021 / \mathrm{cr} 2001178$.

[26] N. Khlebtsov and L. Dykman, "Biodistribution and toxicity of engineered gold nanoparticles: A review of in vitro and in vivo studies," Chem. Soc. Rev., vol. 40, no. 3, pp. 1647-1671, 2011, doi: $10.1039 / \mathrm{c} 0 \mathrm{cs} 00018 \mathrm{c}$.

[27] M. A. Paun, J. M. Sallese, and M. Kayal, "Hall effect sensors design, integration and behavior analysis," J. Sens. Actuator Networks, vol. 2, no. 1, pp. 85-97, 2013, doi: 10.3390/jsan2010085.

[28] M. A. Paun, J. M. Sallese, and M. Kayal, "Comparative study on the performance of five different hall effect devices," Sensors (Switzerland), vol. 13, no. 2, pp. 2093-2112, 2013, doi: $10.3390 / \mathrm{s} 130202093$.

[29] V. P. Kunets et al., "InSb quantum-well-based micro-hall devices: Potential for pT Detectivity," IEEE Trans. Electron Devices, vol. 56, no. 4, pp. 683-687, 2009, doi: 10.1109/TED.2009.2014187.

[30] N. Haned and M. Missous, "Nano-tesla magnetic field magnetometry using an InGaAsAlGaAs-GaAs 2DEG Hall sensor," Sensors Actuators, A Phys., vol. 102, no. 3, pp. 216-222, 2003, doi: 10.1016/S0924-4247(02)00386-2.

[31] L. Huang, Z. Zhang, B. Chen, X. Ma, H. Zhong, and L. M. Peng, "Ultra-sensitive graphene Hall elements," Appl. Phys. Lett., vol. 104, no. 18, 2014, doi: 10.1063/1.4875597. 